EFI 92-09

\title{
Exceptional Superconformal Algebras
}

\author{
P. BOWCOCK ${ }^{12}$ \\ Enrico Fermi Institute, University of Chicago, Chicago, IL 60637, U.S.A.
}

\begin{abstract}
Reductive $\mathrm{W}$-algebras which are generated by bosonic fields of spin-1, a single spin-2 field and fermionic fields of spin-3/2 are classified. Three new cases are found: a 'symplectic' family of superconformal algebras which are extended by $s u(2) \oplus s p(n)$, an $N=7$ and an $N=8$ superconformal algebra. The exceptional cases can be viewed as arising a Drinfeld-Sokolov type reduction of the exceptional Lie superalgebras $F(4)$ and $G(3)$, and have an octonionic description. The quantum versions of the superconformal algebras are constructed explicitly in all three cases.
\end{abstract}

Feb. 1992

\footnotetext{
${ }^{1}$ Email Bowcock@rabi.uchicago .edu

${ }^{2}$ Supported by U.S. DOE grant DEFG02-90-ER-40560 and NSF grant PHY900036
} 


\section{Introduction}

The construction of superconformal algebras has a long history [1,2]. Because of their easy applicability to string theory, the first such algebras were linear and contained one spin-2 field $L(z), N$ spin-3/2 fields $G^{i}(z)$, a number of free fermions $\psi^{j}(z)$ and spin-1 currents $T^{a}(z)$. The linearity of the algebra ensured the existence of a finite dimensional Lie su-

peralgebra consisting of the modes $L_{-1}, L_{0}, L_{1}, G_{-1 / 2}^{i}, G_{1 / 2}^{i}, T_{0}^{a}$. Since simple superalgebras have been classified, this enabled the authors of [2] to list various possibilities. For a long time it was thought that this list was complete, until a new $N=4$ algebra was constructed $[3,4]$ based on a non-simple superalgebra.

Besides these linear algebras, two families of non-linear superconformal algebras exist for arbitrary $N[5-7]$. As $\mathrm{W}$-algebras, these are generated by fields of spin-1,3/2 and one field of spin- 2 but the anticommutator of two supercharges contains a piece quadratic in the currents

$$
\left\{G_{r}^{i}, G_{s}^{j}\right\}=\ldots .+\pi_{i j \times}^{a b} \times T^{a} T_{\times}^{b \times} \times
$$

where ${ }_{\times}^{\times} T^{a} T_{\times}^{b \times}$ is the normal ordered product of two currents, and $\pi$ is symmetric in its upper and lower indices. The vacuum preserving algebra generated by the modes $L_{-1}, L_{0}, L_{1}, G_{-1 / 2}, G_{1 / 2}, T_{0}^{a}$ is not finite because of the non-linearity of the algebra, but can be made so by taking the limit in which the central charge $c \rightarrow \pm \infty[8]$. In this way we can again utilise Lie superalgebra theory to classify algebras which are associative in this limit. For instance the two known families of non-linear algebras correspond to $\operatorname{csp}(N, 2)$ and $s u(N \mid 2)$, or in the notation of Kac [9], $D(N, 1), B(N, 1)$ and $A(N, 1)$. Furthermore, these algebras arise from a Drinfeld-Sokolov reduction [10] of the affine versions of these superalgebras.

It is natural to ask whether or not we can construct any other superconformal algebras. In particular, the $N=1,2$ and 4 algebras can be given a Kazama-Suzuki construction [11] using symmetric, hermitian symmetric, and quaternionic spaces [12], and this suggests a natural association with the reals, complex numbers and quaternions, and in turn suggests the existence of octonionic analogues for these algebras. Further evidence is provided by the existence of an octonionic string solution to low energy heterotic string theory [13]. It is tempting to conjecture the existence of some sort of octonionic superconformal world-sheet symmetry for the effective action of this solution.

In this paper we first classify the possible field contents of superconformal algebras using the results of [8] and [9]. We do indeed find two octonionic superconformal algebras based on the exceptional superalgebras $F(4)$ and $G(3)$ [14,15], and also new is a third infinite family extended by $s u(2) \oplus s p(N)$. The main part of this paper is the explicit construction of the quantum version of each of these algebras by solving Jacobi's identity. 
The structure constants of both $F(4)$ and $G(3)$, and thus of the $N=7$ and $N=8$ algebras that we construct, can be naturally expressed in terms of octonions [16]. It seems likely that the $N=7$ and $N=8$ algebras are an octonionic generalisation of the $N=3$ and $N=4$ algebras respectively. Unfortunately, we find that the symplectically extended superconformal algebras cannot have unitary highest weight representations.

We conclude with some remarks on future directions of research, and in particular on the possibility of finding a linear version of these algebras.

\section{Classification of superconformal algebras}

In this section we shall classify $W$-algebras with the following properties. We assume that the bosonic generators of the algebra consist of a number of spin-1 fields and a spin-2 field forming the semi-direct product of a Kac-Moody algebra with a Virasoro algebra. The fermionic generators are all of spin-3/2. We exclude the possibility of spin- $1 / 2$ generators.

We further demand that the algebra be reductive [8]. This essentially means that the algebra exists in the limit that the central charge $c \rightarrow \infty$ and that the classical analogue of the algebra can be recovered in this limit. In this case this implies that there is a coefficient in front of the quadratic piece of (1.1) which vanishes in this limit, so that the algebra linearises. In [8] it was demonstrated that such algebras were characterised by pairs $(g, h)$ where $g$ is simple (super)algebra and $h$ is an $s u(2)$ subalgebra of $g$. The number, statistics and conformal dimension of generators of the corresponding $W$-algebras are given by the number, grading and spins of irreducible representations in the decomposition of $g$ with respect to $h$. Each irreducible representation of spin $j$ corresponds to a primary generator of conformal dimension $j+1$, and bosonic(fermionic) generators correspond to representations in the even(odd) parts of the superalgebra.

Denote the even and odd parts of $g$ by $g_{E}$ and $g_{O}$. The requirement that the algebra be superconformal, that is have the field content alluded to above, means that $g_{E}$ should decompose into a number of spin-0 and one spin- 2 representation, and $g_{O}$ into a number of spin-1/2 representations under the decomposition with respect to $h$. In particular, this implies that $g_{E}=h \oplus h^{\prime}$ and that $g_{O}$ transforms as a $(1 / 2, \Lambda)$ with respect to $h \oplus h^{\prime}$, where $\Lambda$ is some representation of $h^{\prime}$.

In table 1, we enumerate the possibilities, using the classification of simple Lie superalgebras [9]. In the first column we list the simple superalgebras. The corresponding even graded algebra $g_{E}$ is given in the second column while the next column shows how $g_{O}$ transforms under $g_{E}$. In the last three columns we list the choices of $h$ which yield a superconformal algebra, the number of supersymmetry generators and the Kac-Moody component of the resulting algebra. We do not include the superalgebras $P(n), Q(n), W(n), S(n), \tilde{S}(n)$ or $H(n)$ since these do not yield superconformal algebras, except in the special cases that 
they are isomorphic to a superalgebra already considered. There is no need to consider semi-simple algebras, since if $h$ is embedded non-trivially in more than one ideal of $g$ then it is easy to see that the resulting algebra contains more than one spin- 2 field. If, on the other hand, $h$ is a subalgebra of some simple ideal in $g$, then the resulting W-algebra is simply a direct product of the $\mathrm{W}$-algebra associated with the reduction of this ideal and a Kac-Moody algebra. It is possible to couple these two parts non-trivially using fields of less than spin-1 such as free fermions, but this case has been excluded by our assumptions. This possibility can lead to interesting algebras, as in the case of the linear $N=4$ algebra, and we return to this point in our conclusions.

\begin{tabular}{|c|c|c|c|c|c|}
\hline$g$ & $g_{E}$ & $g_{O}$ & $(m, n)$ & $N$ & KM \\
\hline$A(m, m)$ & $A_{m} \oplus A_{m}$ & $(m+1, m+1)$ & $m=1$ & 4 & $A_{1}$ \\
$A(m, n)$ & $u(1) \oplus A_{n+1}$ & $(2 m+2, n+1)$ & $n=1$ & $2(m+1)$ & $u(m+1)$ \\
$B(m, n)$ & $B_{m} \oplus C_{n}$ & $(2 m+1,2 n)$ & $n=1$ & $2 m+1$ & $B_{m}$ \\
$D(m, n)$ & $D_{m} \oplus C_{n}$ & $(2 m, n)$ & $n=1$ & $2 m$ & $D_{m}$ \\
& & & $m=2$ & $4 m$ & $A_{1} \oplus C_{n}$ \\
$C(n)$ & $u(1) \oplus C_{n}$ & $(-, n)$ & $n=1$ & 2 & $u(1)$ \\
$D(2,1, \alpha)$ & $A_{1} \oplus A_{1} \oplus A_{1}$ & $(2,2,2)$ & & 4 & $A_{1} \oplus A_{1}$ \\
$F(4)$ & $A_{1} \oplus B_{3}$ & $(2,8)$ & & 8 & $B_{3}$ \\
$G(3)$ & $A_{1} \oplus G_{2}$ & $(2,7)$ & & 7 & $G_{2}$ \\
\hline
\end{tabular}

Table 1

One readily identifies $N=1$ and $N=2$ superconformal algebras with $B(0,1), A(0,1) \equiv$ $C(1) \equiv D(1,1)$, the $N=3$ algebra with $B(1,1)$, the $N=4$ algebra extended by $s u(2)$ with $A(1,1)$, and extended by $s u(2) \oplus s u(2)$ with $D(2,1, \alpha)$. As mentioned above, the two infinite families of Knizhnik and Bershadsky can be identified with $A(m, 1)$ for $m>1$ and with $B(m, 1), m>1$ and $D(m, 1), m>2$. There are three further possibilities: an infinite family of $s u(2) \oplus s p(m)$ extended superconformal algebras corresponding to $D(2, m)$, and two exceptional superconformal algebras corresponding to the exceptional superalgebras $G(3)$ and $F(4)[17]$. For these algebras one finds that $g_{E}=s u(2) \oplus g_{2}, s u(2) \oplus s o(7)$ respectively, and $g_{O}$ transforms under $g_{E}$ as a $(1 / 2,7),(1 / 2,8)$ respectively. Thus choosing $h$ to be the $s u(2)$ factor of $g_{E}$, we expect that there exists an $N=7$ superconformal algebra with $\hat{g}_{2}$ as a subalgebra associated with $G(3)$, and an $N=8$ superconformal algebra with $\hat{s o}(7)$ as a subalgebra associated with $F(4)$. 
Though in general it is not known whether the algebra associated to a particular pair $(g, h)$ is unique, in the next section we shall explicitly demonstrate that the above algebras are completely determined by their field content and associativity, proofing the completeness of our classification of reductive superalgebras generated by fields of spin-1, spin-3/2 and spin-2.

\section{The commutation relations of the $\mathrm{N}=7, \mathrm{~N}=8$ and symplectic superconformal algebras}

In what follows we derive the commutation relations for the three new superconformal algebras based on $G(3), F(4)$ and $D(2, N)$. This simply amounts to writing down the general form of the commutation relations and checking Jacobi's identity. The bosonic part of the algebra consists of the semi-direct product of a Kac-Moody algebra with the Virasoro algebra:

$$
\begin{aligned}
{\left[T_{m}^{a}, T_{n}^{b}\right] } & =-f^{a b c} T_{m+n}^{c}+k m \delta_{m+n, 0} \\
{\left[L_{m}, L_{n}\right] } & =(m-n) L_{m+n}+\frac{c}{12} m\left(m^{2}-1\right) \delta_{m+n, 0} \\
{\left[L_{m}, T_{n}^{a}\right] } & =-n T_{m+n}^{a}
\end{aligned}
$$

where $f^{a b c}$ are the structure constants of $g_{2}, s o(7)$ and $s u(2) \oplus s p(N)$ for the $N=7, N=8$ and symplectic series of algebras respectively, normalised so that the length of a long root squared is two. Jacobi's identity is easily verified for these generators. The superconformal generators $G_{r}^{i}$ are highest weight both for the Kac-Moody algebra and the Virasoro algebra; that is

$$
\begin{aligned}
{\left[L_{m}, G_{r}^{i}\right] } & =\left[\frac{m}{2}-r\right] G_{m+r}^{i} \\
{\left[T_{m}^{a}, G_{r}^{i}\right] } & =M_{i j}^{a} G_{m+r}^{j}
\end{aligned}
$$

where $M_{i j}^{a}$ is the seven-dimensional representation of $g_{2}$, the eight-dimensional spinor representation of $s o(7)$, or the $(2,2 N)$ representation of $s u(2) \oplus \operatorname{sp}(N)$ for the three cases respectively, and satisfies

$$
\left[M^{a}, M^{b}\right]=f^{a b c} M^{c} .
$$

With these commutation relations it is easy to see that the TTG and LLG Jacobi constraints are satisfied. The only remaining equations that a consistent algebra must satisfy come from the combinations $T G G, L G G$ and $G G G$. To check the first two relations one can exploit Virasoro and Kac-Moody Ward identities. If we write the operator product expansion of two superconformal generators as

$$
G^{i}(z) G^{j}(\zeta)=\sum_{n \geq 0} \psi_{n}^{i j}(\zeta)(z-\zeta)^{-3+n}
$$


then these identities are respectively

$$
\begin{aligned}
L_{m}\left|\psi_{n}^{i j}\right\rangle & =(n+m / 2-3 / 2)\left|\psi_{n-m}^{i j}\right\rangle \\
T_{m}^{a}\left|\psi_{n}^{i j}\right\rangle & =M_{i k}^{a}\left|\psi_{n-m}^{k j}\right\rangle,
\end{aligned}
$$

where $n>0$ and the state $|\psi\rangle$ is given by the usual correspondence $|\psi\rangle=\lim _{z \rightarrow 0} \psi(z)|v a c\rangle$. Once these two relations have been satisfied, it only remains to check the $G G G$ Jacobi identity. We now deal with each case in turn. The calculation is somewhat involved, and a number of relevant identities and conventions are given in an appendix.

\subsection{The $N=7$ SUPERALGEBRA}

Writing out explicitly the operator product expansion (3.7) in modes and in a basis of primary fields we find that the most general form for the anticommutation relation of two superconformal generators can be written

$$
\begin{aligned}
\left\{G_{r}^{i}, G_{s}^{j}\right\} & =\frac{c}{3}\left(r^{2}-\frac{1}{4}\right) \delta_{r+s, 0}+B \delta^{i j} L_{r+s}+\delta^{i j} D\left(L_{r+s}-\frac{c}{c_{g}} \mathcal{L}_{r+s}\right) \\
& +C \frac{r-s}{2} M_{i j}^{a} T_{r+s}^{a}+E \pi_{i j}^{a b}\left(\underset{\times}{\times} T^{a} T_{\times \times}^{b \times}\right)_{r+s}
\end{aligned}
$$

where $B, C, D, E$ are coefficients to be determined, $\mathcal{L}$ is the Sugawara energy momentum tensor

$$
\mathcal{L}_{m}=\frac{1}{2(k+4)}\left(\underset{\times}{\times} T^{a} T_{\times}^{a}\right)_{m}
$$

for $g_{2}$ whose central charge is given by $c_{g}=14 k /(k+4)$. The normalisation of $G$ has been fixed by the first term in (3.10). The tensor $\pi_{i j}^{a b}$ is symmetric and traceless in both upper and lower indices. The Virasoro Ward identity (3.8) gives that $B=2$. The Kac-Moody Ward identity implies that $C=2 c / 3 k$ and yields the equation

$$
\begin{aligned}
\frac{2 c}{3 k} M_{i x}^{a} M_{x j}^{b} & =\frac{c}{3 k} f^{a b c} M_{i j}^{c}+\delta^{a b} \delta_{i j}\left[2+D\left(1-\frac{c}{c_{g}}\right)\right] \\
& +\pi_{i j}^{e f}\left[2 k \delta^{e a} \delta^{f b}-f^{y a e} f^{y b f}\right]
\end{aligned}
$$

The matrix $M^{a}$ satisfies the properties

$$
\begin{aligned}
\operatorname{Tr}\left(M^{a} M^{b}\right) & =2 \delta^{a b} \\
\sum_{a} M_{i j}^{a} M_{k l}^{a} & =\frac{2}{3}\left(\delta_{j k} \delta_{i l}-\delta_{i k} \delta_{j l}\right)+1 / 3 \eta_{i j k l}
\end{aligned}
$$

where the $\eta$ is a totally antisymmetric matrix best defined in terms of the octonion structure constants $c_{i j k}$ as

$$
c_{x i j} c_{x k l}=\eta_{i j k l}+\delta_{i k} \delta_{j l}-\delta_{i l} \delta_{j k}
$$


For a particular choice for the values of $c_{i j k}, \eta_{i j k l}$, and $M$ we refer the reader to the appendix and to [14]. Taking the trace of (3.12) we find immediately that

$$
2+D\left(1-\frac{c}{c_{g}}\right)=\frac{4 c}{21 k}
$$

The traceless symmetric part of (3.12) is solved by setting

$$
\begin{aligned}
\pi_{i j}^{a b} & =\left(M^{a} M^{b}+M^{b} M^{a}\right)_{i j}-\frac{4}{7} \delta^{a b} \delta_{i j} \\
E \frac{6 k+10}{3} & =\frac{c}{3 k}
\end{aligned}
$$

where we have made extensive use of (3.14).

Our only remaining task is to ensure that the Jacobi identity for three superconformal generators is satisfied. Noting that

$$
\begin{aligned}
{\left[{ }_{\times}^{\times} T^{a} T_{\times m}^{b \times}, G_{r}^{i}\right] } & =\frac{1}{3}\left(\frac{m}{2}-r\right)\left(M^{a} M^{b}+M^{b} M^{a}\right)_{i j} G_{m+r}^{j} \\
& -(m+1) f^{a b c} M_{i j}^{c} G_{m+r}^{j}+M_{i j \times}^{a} T^{b} G_{\times m+r}^{j \times}+M_{i j \times}^{b \times} T^{a} G_{\times m+r}^{j \times}
\end{aligned}
$$

and using a number of other identities (again in the appendix) we find two further equations

$$
\begin{aligned}
\frac{32 E}{21} & =\frac{c D}{c_{g}(k+4)} \\
2+D\left(1-\frac{4 c}{3 c_{g}(k+4)}\right) & =\frac{4 c}{9 k}+\frac{32 E}{21}
\end{aligned}
$$

Equations (3.16),(3.18),(3.20) and (3.21) contain the four variables $D, E, k$ and $c$. This is in seeming contradiction to the assumption that this algebra is reductive; that is to say it is associative for continuous $c$. Thus we expect that the value of $c$ is not fixed and parameterises the solution. It is a good check on our calculation that indeed the four equations are not independent, and admit the following one parameter family of solutions

$$
\begin{aligned}
c & =\frac{k(9 k+31)}{2(k+3)} \\
E & =\frac{c}{2 k(3 k+5)} \\
D & =\frac{32}{3(3 k+5)}
\end{aligned}
$$

As mentioned earlier, we expect that $W$-algebras of this type linearise in the limit that $c \rightarrow \infty$. In this limit $c$ is proportional to $k$ and we note that the coefficients $D, E$ of the quadratic terms in (3.10) tend to zero, confirming our expectations. 
The superalgebra $G(3)$ has an octonionic description in which the bosonic part is viewed as the direct sum of $s u(2)$ and the derivation algebra $g_{2}$ of the octonions. The fermionic part can then be represented as a doublet of pure imaginary octonions [16]. The quaternionic version of this algebra has the direct product of an $s u(2)$ with the derivation algebra of the quaternions which is another copy of $s u(2)$ as its bosonic part, acting on a doublet of the three pure imaginary quaternions. This is the superalgebra $B(1,1)$ in Kac's notation which is the superalgebra associated with the $N=3$ superconformal algebra. Similarly we can associate the Virasoro algebra and the $N=1$ superconformal algebra with the reals and the complex numbers.

\subsection{The $N=8$ SUPERCONFORMAL ALGEBRA}

The calculation for the $N=8$ superconformal algebra is very similar to those sketched above. It is more convenient to label the adjoint representation of $s o(7)$ by a pair of antisymmetric indices $I, J=1 \ldots 7$, so that the commutation relations of the corresponding Kac-Moody algebra are

$$
\begin{aligned}
{\left[T_{m}^{I J}, T_{n}^{K L}\right] } & =-i\left(\delta_{J K} \delta_{I M} \delta_{L N}-\delta_{I K} \delta_{J M} \delta_{L N}-\delta_{J L} \delta_{I M} \delta_{K N}+\delta_{I L} \delta_{J M} \delta_{K N}\right) T_{m+n}^{M N} \\
& +k m\left(\delta^{I K} \delta^{J L}-\delta^{I L} \delta^{J K}\right)
\end{aligned}
$$

Since we are interested in the spinor representation of $s o(7)$ we introduce gamma matrices which can be written [18]

$$
\left(\gamma^{I}\right)_{i j}=i\left(c_{I i j}+\delta_{I i} \delta_{j 8}-\delta_{I j} \delta_{i 8}\right)
$$

where $c_{i j k}$ are the structure constants of the octonions as above. We also adopt the normal convention $\gamma^{I J . . N}=\gamma^{[I} \gamma^{J} . . \gamma^{N]}$. The most general form for the commutator of two superconformal generators is given by

$$
\begin{aligned}
& \left\{G_{r}^{i}, G_{s}^{j}\right\}=\frac{c}{3}\left(r^{2}-\frac{1}{4}\right) \delta_{r+s, 0}+B \delta^{i j} L_{r+s}+\delta^{i j} D\left(L_{r+s}-\frac{c}{c_{g}} \mathcal{L}_{r+s}\right) \\
& +C \frac{r-s}{2} \gamma_{i j}^{I J} T_{r+s}^{I J}+E \gamma_{i j}^{I J K L}\left(\underset{\times}{\times} T^{I J} T_{\underset{\times}{K})_{r+s}}^{K L}\right.
\end{aligned}
$$

The Virasoro and Kac-Moody Ward identities give that

$$
\begin{aligned}
B & =2 \\
C & =\frac{i c}{6 k} \\
2+D\left(1-\frac{c}{c_{g}}\right) & =\frac{c}{6 k} \\
E & =-\frac{c}{48 k(k+2)}
\end{aligned}
$$


The Jacobi identity for three superconformal generators gives us the two equations

$$
\begin{aligned}
\frac{c D}{c_{g}(4 k+20)} & =-4 E \\
2+D\left(1-\frac{c}{c_{g}(4 k+20)}\right)-\frac{c}{2 k}+20 E & =0 .
\end{aligned}
$$

Again we find that the four equations (3.30),(3.31), (3.32) and (3.33) are not independent and have the solution

$$
\begin{aligned}
c & =\frac{2 k(2 k+11)}{(k+4)} \\
D & =\frac{7}{(k+2)} \\
E & =-\frac{c}{48 k(k+2)} .
\end{aligned}
$$

As in the $N=7$ case, this algebra has a natural description in terms of the structure constants of the octonions. It is not entirely obvious what the quaternionic analogue of this algebra is. The most naive guess might be an algebra with four supercharges transforming under the spinor and complex conjugate spinor representations of so(3), or in other words, the superconformal algebra of [1]. On the other hand one can introduce an extra so(3) into the quaternionic analogy by considering the group of similarity triples $[16,20]$, rather than the group of norm preserving transformations, suggesting that we should consider the non-linear $\tilde{A}_{\gamma}$ algebra as the quaternionic equivalent.

\subsection{The SympleCtic SUPERCONFORMAL ALGEBraS}

Corresponding to the superalgebras $D(2, N)$ there exists a family of superconformal algebras with $4 N$ fermionic generators. The algebra contains an $s u(2) \oplus s p(N)$ Kac-Moody component, under which the fermionic generators transform as a $(2,2 N)$ representation. We label $s p(n)$ adjoint indices by $x, y \ldots$ and $s u(2)$ adjoint indices by $a, b \ldots$. We label the $4 N$-dimensional representation space by Greek letters $\alpha, \beta \ldots$ although it will be convenient to decompose these indices into a pair of indices $(i, I)$ where $i=1, \ldots 4$ and $I=1, \ldots N$. Thus we write

$$
\begin{aligned}
& {\left[T_{m}^{a}, G_{r}^{\alpha}\right]=M_{\alpha \beta}^{a} G_{m+r}^{\beta}} \\
& {\left[T_{m}^{x}, G_{r}^{\alpha}\right]=M_{\alpha \beta}^{x} G_{m+r}^{\beta}}
\end{aligned}
$$

where we can take the generators of $s u(2)$ to be $\frac{i}{\sqrt{2}} \gamma_{i j}^{+a} \delta_{I J}$ and the generators of $s p(N)$ can be taken to be $\frac{i}{\sqrt{2}} \gamma_{\alpha \beta}^{-a} \delta_{A I} \delta_{A J}$ for $A=1 . . N, i \gamma_{\alpha \beta}^{-a}\left(\delta_{A I} \delta_{B J}-\delta_{A J} \delta_{B J}\right)$ and $\frac{i}{2} \delta_{\alpha \beta}\left(\delta_{A I} \delta_{B J}-\right.$ 
$\left.\delta_{A J} \delta_{B J}\right)$.Here we can take

$$
\gamma_{\alpha \beta}^{a \pm}=\epsilon_{a \alpha \beta} \pm\left(\delta_{a \alpha} \delta_{\beta 4}-\delta_{a \beta} \delta_{\alpha 4}\right) .
$$

Note that this is simply the quaternionic analogue of the formula (3.26). With this choice $\operatorname{tr}\left(M^{a} M^{b}\right)=2 N \delta^{a b}, \operatorname{tr}\left(M^{a} M^{x}\right)=0$ and $\operatorname{tr}\left(M^{x} M^{y}\right)=2 \delta^{x y}$. Unlike the previous examples, the Kac-Moody part of the algebra is the direct sum of two simple pieces, and so the most general form of the commutation relations contain the central charge and two levels $k, k^{\prime}$ where

$$
\begin{aligned}
{\left[T_{m}^{a}, T_{n}^{b}\right] } & =-f^{a b c} T_{m+n}^{c}+k m \delta^{a b} \delta_{m+n, 0} \\
{\left[T_{m}^{x}, T_{n}^{y}\right] } & =-f^{x y z} T_{m+n}^{z}+k^{\prime} m \delta^{x y} \delta_{m+n, 0}
\end{aligned}
$$

and we have normalised the currents so that $\left[M^{a}, M^{b}\right]=f^{a b c} M^{c}$ etc. as before.

The anticommutator of two fermionic generators can be written as

$$
\begin{aligned}
\left\{G_{r}^{\alpha}, G_{s}^{\beta}\right\} & =\frac{c}{3}\left(r^{2}-\frac{1}{4}\right) \delta_{r+s, 0}+2 \delta^{\alpha \beta} L_{r+s} \\
& +\delta^{\alpha \beta} D\left(L_{r+s}-\frac{c}{c_{g}} \mathcal{L}^{s u(2)} B_{r+s}\right)+D^{\prime}\left(L_{r+s}-\frac{c}{c_{g}^{\prime}} \mathcal{L}_{r+s}^{s p(n)}\right) \\
& +\frac{r-s}{2}\left(\frac{2 c}{3 k} M_{\alpha \beta}^{a} T_{r+s}^{a}+\frac{2 c}{3 k^{\prime}} M_{\alpha \beta}^{x} T_{r+s}^{x}\right) \\
& +E \pi_{\alpha \beta}^{a x}\left(\underset{\times}{\times} T^{a} T_{\times}^{x}\right)_{r+s}+E^{\prime} \pi_{\alpha \beta}^{x y}\left(\begin{array}{r}
\times \\
\times
\end{array} T^{x} T_{\times}^{y}\right)_{r+s}
\end{aligned}
$$

where

$$
\begin{aligned}
\pi_{\alpha \beta}^{x y} & =\left(M^{a} M^{b}+M^{b} M^{a}\right)_{\alpha \beta}-1 / N \delta^{x y} \delta_{\alpha \beta} \\
\pi_{\alpha \beta}^{a b} & =\left(M^{x} M^{y}+M^{y} M^{x}\right)_{\alpha \beta} \\
c_{g} & =\frac{3 k}{k+2} \\
c_{g^{\prime}} & =\frac{N(2 N+1) k^{\prime}}{k^{\prime}+N+1} .
\end{aligned}
$$

Note that there is no term containing the symmetric traceless product of currents ${ }_{\times}^{\times} T^{a} T^{b \times}$. This is because such a product transforms as spin-2 multiplet under $s u(2)$ and there is no invariant way to couple this to the pair of spin- $1 / 2$ indices $\alpha, \beta$.

Demanding associativity yields the following equations:

$$
\begin{aligned}
E & =\frac{c}{3 k k^{\prime}} \\
E^{\prime} & =\frac{c}{3 k^{\prime}\left(2 k^{\prime}+N+2\right)}
\end{aligned}
$$




$$
\begin{aligned}
2+D\left(1-\frac{c}{c_{g}}\right)+D^{\prime} & =\frac{c}{3 k} \\
2+D^{\prime}\left(1-\frac{c}{c_{g^{\prime}}}\right)+D & =\frac{c}{3 k^{\prime} N} \\
E^{\prime} \frac{N+1}{4}-\frac{E}{4} & =\frac{c}{6 k}+\frac{c}{12 k^{\prime}} \\
\frac{1}{2}\left[2+D+D^{\prime}-\frac{c D}{2 c_{g}(k+2)}\right. & =\frac{c}{12 k^{\prime}}+E^{\prime} \frac{(N+1)(N+2)}{12 N}+\frac{E}{4} \\
\left.-\frac{c D^{\prime}(2 N+1)}{6 c_{g^{\prime}}\left(k^{\prime}+N+1\right)}\right] & =-E^{\prime} \\
\frac{c D^{\prime}}{c_{g^{\prime}}\left(k^{\prime}+N+1\right)} & =E^{\prime} \frac{(N-2)}{N} \\
\frac{c D}{c_{g}(k+2)} & =E .
\end{aligned}
$$

From these it is easy to show that the levels $k, k^{\prime}$ are related by the equation

$$
2 k^{\prime}+k+N+2=0
$$

The remaining variables are given by

$$
\begin{aligned}
D & =\frac{1}{k^{\prime}} \\
D^{\prime} & =\frac{-(N-2)(2 N+1)}{3 k} \\
E & =-E^{\prime}=\frac{c}{3 k k^{\prime}} \\
c & =\frac{6 k k^{\prime}+3 k-k^{\prime}(N-2)(2 N+1)}{k+k^{\prime}+2} .
\end{aligned}
$$

As a check we note that for $N=1$ this algebra should coincide with the known nonlinear $N=4$ algebra, $\tilde{A}_{\gamma}$. The formula for the central charge of $\tilde{A}_{\gamma}$ is

$$
c=\frac{6(k+1)\left(k^{\prime}+1\right)}{k+k^{\prime}+2}-3
$$

which coincides with (3.61) for $N=1$. The formulae for the coefficients $D, D^{\prime}$ are only correct if we use the relation between the levels (3.57) which is not needed for associativity

of the $\tilde{A}_{\gamma}$ algebra. In fact one needs to rederive the associativity equations in this case since a number of special relations hold. For instance, the tensor $\pi^{x y}$ identically vanishes. coefficient is $E^{\prime}$ vanishes Unfortunately, for $N>1$ there is no possibility that this algebra can have unitary highest weight representations since (3.57) does not allow $k$ and $k^{\prime}$ to be positive at the same time, which is a requirement for such representations. 


\section{Conclusions}

We have succeeded in constructing three new non-linear quantum superconformal algebras, based on the Lie superalgebras $F(4), G(3)$ and $D(2, m)$. One can easily recover the classical versions of these algebras by making the substitutions

$$
L^{\prime}=\hbar L, G^{i}=\hbar G^{i}, T^{\prime a}=\hbar T^{a}, c^{\prime}=\hbar c
$$

and calculating the Poisson brackets of primed quantities using the usual correspondence; e.g.

$$
\left\{G^{\prime i}, G^{\prime j}\right\}_{\text {P.B }}=\lim _{\hbar \rightarrow 0}\left\{G^{\prime i}, G^{\prime j}\right\}
$$

while keeping $c^{\prime}$ fixed.

The symplectic series of superconformal algebras do not seem useful, at least in the context of rational conformal field theory, since they do not admit highest weight unitary representations. The $N=7$ and $N=8$ superalgebras have no obvious obstruction to having such representations however and it should be interesting to develop a representation theory for these algebras. We also need to make the analogy between these algebras and the non-linear $N=3$ and $N=4$ algebras more precise. The latter can be constructed using a Kazama-Suzuki construction on quaternionic(Wolf) spaces [12]. It would be interesting to see whether some corresponding construction exists for the new $N=8$ algebra, and what space one would need to consider in such a construction. The free field realisation of these algebras which arises from a generalised Drinfeld-Sokolov reduction of the superalgebras $F(4)$ and $G(3)$ may be related to the above questions.

The classification we give is only complete if we restrict ourselves to extending the Virasoro algebra by fields of spin- 1 and spin $3 / 2$. For instance, both the $N=3$ and the $N=4$ algebras exist in linear and non-linear forms, so that we may hope that the same is true of their octonionic versions. It is known how to systematically remove the free fermions from a linear algebra to obtain a fermion-free and generically non-linear algebra [21]. However, it is not clear how to implement the reverse procedure, coupling the non-linear algebras to a number of free fermions which may interact further with a new Kac-Moody algebra to obtain a linear algebra. It remains an open question whether this can be done in the present case. The analysis of [22] does not include the possibility of spin- $1 / 2$ fermions, and though the authors of [2] include fermions, the situation where these are coupled to another Kac-Moody algebra is not considered. We hope to settle this question in the near future. 


\section{Appendix}

The structure constants $c_{i j k}$ can be taken to be totally antisymmetric. We shall use the notation of [14] and so $c_{i j k}$ takes the value one for any of the triples

$$
(1,2,3),(1,4,5),(1,7,6),(2,4,6),(2,5,7),(3,4,7),(3,6,5) \text {. }
$$

For this choice the tensor $\eta_{i j k l}$ is non-vanishing for permutations of

$$
\begin{array}{r}
(1,2,4,7),(1,2,6,5),(1,3,6,4),(1,3,5,7) \\
(2,3,4,5),(2,3,7,6),(4,5,7,6) .
\end{array}
$$

The relation (3.14) can be proved by considering the following embedding of $g_{2} \in s o(7)$ :

$$
\begin{array}{rlrl}
M^{1} & =\frac{1}{\sqrt{2}}\left(T^{41}+T^{36}\right) & M^{2}=\frac{1}{\sqrt{6}}\left(T^{41}-T^{36}-2 T^{27}\right) \\
M^{3}=\frac{1}{\sqrt{2}}\left(T^{31}-T^{46}\right) & M^{4}=\frac{1}{\sqrt{6}}\left(T^{31}+T^{46}-2 T^{57}\right) \\
M^{5}=\frac{1}{\sqrt{2}}\left(T^{21}-T^{56}\right) & M^{6}=\frac{1}{\sqrt{6}}\left(T^{21}+T^{56}+2 T^{47}\right) \\
M^{7}=\frac{1}{\sqrt{2}}\left(T^{51}+T^{26}\right) & M^{8}=\frac{1}{\sqrt{6}}\left(T^{51}-T^{26}+2 T^{37}\right) \\
M^{9}=\frac{1}{\sqrt{2}}\left(T^{24}-T^{53}\right) & M^{1} 0=\frac{1}{\sqrt{6}}\left(T^{24}+T^{53}-2 T^{17}\right) \\
M^{11}=\frac{1}{\sqrt{2}}\left(T^{54}+T^{23}\right) & M^{12}=\frac{1}{\sqrt{6}}\left(T^{54}-T^{23}-2 T^{67}\right) \\
M^{13}=\frac{1}{\sqrt{2}}\left(T^{43}-T^{16}\right) & M^{14}=\frac{1}{\sqrt{6}}\left(T^{43}+T^{16}+2 T^{25}\right)
\end{array}
$$

where the matrix $T^{I J}$ can be taken to be the vector representation

$$
T_{i j}^{I J}=-i\left(\delta_{I i} \delta_{J j}-\delta_{I j} \delta_{J i}\right)
$$

The matrix $\pi_{i j}^{a b}$ satisfies the equations

$$
\begin{aligned}
\sum_{\text {cyclic } i j k} \pi_{i j}^{a b} M_{k l}^{a} & =\frac{16}{21} \sum_{\text {cyclic } i j k} \delta_{i j} M_{k l}^{b} \\
\pi_{i j}^{a b} \pi_{k l}^{a b} & =\frac{32}{9}\left(\delta_{i k} \delta_{j l}+\delta_{j k} \delta_{i l}-\frac{2}{7} \delta_{i j} \delta_{k l}\right)
\end{aligned}
$$

For the $N=8$ case it is useful to extend the tensor $\eta_{i j k l}$ to the totally antisymmetric tensor $[18,19]$

$$
c_{a b c d}^{ \pm}=\eta_{a b c d} \mp\left(c_{a b c} \delta_{d 8}+c_{b c d} \delta_{a 8}+c_{c d a} \delta_{b 8}+c_{d a b} \delta_{c 8}\right)
$$


where now the indices $a, b, c, d$ run from 1 to 8 . One can establish that

$$
\begin{aligned}
\gamma_{a b}^{i j} & =c_{i j a b}^{+}+\delta_{i a} \delta_{j b}-\delta_{i b} \delta_{j a} \\
\sum_{i j=1}^{7} \gamma_{a b}^{i j} \gamma_{c d}^{i j} & =6\left(\delta_{a c} \delta_{b d}-\delta_{a d} \delta_{b c}\right)-2 c_{a b c d}^{-} \\
\sum_{i j=1}^{7} \gamma_{a b}^{i j k l} \gamma_{c d}^{i j} & =3 \delta_{a c} \gamma_{d b}^{k l}-3 \delta_{a d} \gamma_{c b}^{k l}+3 \delta_{b d} \gamma_{a c}^{k l}-3 \delta_{b c} \gamma_{a d}^{k l} \\
& +2 \delta_{a b} \gamma_{c d}^{k l}+c_{a x c d}^{-} \gamma_{b x}^{k l}+c_{b x c d}^{-} \gamma_{a x}^{k l} \\
\gamma_{a b}^{i j k l} \gamma_{c d}^{i j k l} & =96\left(\delta_{a c} \delta_{b d}-\delta_{b c} \delta_{a d}\right)-24 \delta_{a b} \delta_{c d} .
\end{aligned}
$$

In the symplectic case, most relations needed can be proved using

$$
\begin{aligned}
\sum_{x \in s p(N)} M_{i I j J}^{a} M_{k K l L}^{b}= & -\frac{1}{4} \delta_{i j} \delta_{k l}\left[\delta_{I K} \delta_{J L}-\delta_{I L} \delta_{J K}\right] \\
& -\frac{1}{4}\left[\delta_{i k} \delta_{j l}-\delta_{j k} \delta_{i l}-\epsilon_{i j k l}\right]\left[\delta_{I K} \delta_{J L}+\delta_{I L} \delta_{J K}\right] \\
\sum_{a \in s u(2)} M_{i I j J}^{x} M_{k K l L}^{x}= & -\frac{1}{2}\left[\delta_{i k} \delta_{j l}-\delta_{j k} \delta_{i l}+\epsilon_{i j k l}\right] \delta_{I J} \delta_{K L}
\end{aligned}
$$

The matrices $\pi^{x y}$ and $\pi^{a x}$ satisfy the equations

$$
\begin{aligned}
\sum_{\text {cyclic } \alpha \beta \gamma} 2 \pi_{\alpha \beta}^{x y} M_{\gamma \delta}^{y}-\pi_{\alpha \beta}^{x a} M_{\gamma \delta}^{a} & =\frac{N-2}{N} \sum_{\text {cyclic } \alpha \beta \gamma} \delta_{\alpha \beta} M_{\gamma \delta}^{x} \\
\sum_{\text {cyclic } \alpha \beta \gamma} 2 \pi_{\alpha \beta}^{a y} M_{\gamma \delta}^{y} & =\sum_{\text {cyclic } \alpha \beta \gamma} \delta_{\alpha \beta} M_{\gamma \delta}^{a}
\end{aligned}
$$




\section{References}

[1] M. Ademollo et al. Phys. Lett. B62 (1976) 105.

[2] P. Ramond and J. Schwarz Phys. Lett. B64 (1976) 75.

[3] A. Sevrin, W. Troost and A. van Proeyen Phys Lett. B208 (1988) 447.

[4] M. Gunaydin, J. L. Petersen, A. Taormina and A. van Proeyen Nucl. Phys. B322 (1989) 322.

[5] V. G. Knizhnik Theor. Math. Phys. 66 (1986) 68.

[6] M. Bershadsky Phys. Lett. 174B (1986) 285.

[7] K. Schoutens Nucl. Phys. B295 (1988) 634.

[8] P. Bowcock and G. M. T. Watts, preprint EFI 91-63, DTP 91-63

[9] V. G. Kac Comm. Math. Phys 53 (1977) 31.

[10] V. G. Drinfeld and V. V. Sokolov Dokl. Akad. Nauk. USSR 23 No. 3 (1981).

[11] Y. Kazama and H. Suzuki Phys. Lett. B216 (1989) 112.

[12] A. van Proeyen Class. Quant. Grav 6 (1989) 1501.

[13] J. Harvey and A. Strominger Phys. Rev. Lett. 66 (1991) 549.

[14] M. Scheunert, W. Nahm and V. Rittenberg J. Math. Phys. 17 (1976) 1626.

[15] P. G. O. Freund and I. Kaplansky J. Math. Phys. 17 (1976)

[16] A. Sudbery Journ. Math. Phys. 24 (1983) 1986.

[17] B. de Witt and P. van Nieuwenhuizen Journ. Math. Phys. 23 (1982) 1952.

[18] E. Corrigan, C. Devchand, D. .B .Fairlie and J. Nuyts Nucl. Phys. B214 (1983) 452.

[19] B. de Wit and H. Nicolai Nucl. Phys. B231 (1984) 506.

[20] P. Ramond "Introduction to Exceptional Lie Groups and Algebras" CALT-68-577 (1976)

[21] P. Goddard and A. Schwimmer Phys. Lett. B214 (1988) 209. 
[22] R. Gastmans, A. Sevrin, W. Troost, and A. van Proeyen. Int. J. Mod. Phys A2 (1987) 195.

[23] F. Englert, A. Sevrin, W. Troost, A. van Proeyen, and P. Spindel Journ. Math. Phys 29 (1988) 281. 